\title{
On the Recursion of Syntax and Discourse Structure in Linguistics and the Recursion of Chinese Traditional Landscape Painting
}

\author{
Wenqing Li \\ Integrated Information Department, Zhejiang Art Museum, Hangzhou 310002, Zhejiang, China \\ Email: liwq9294@sina.com
}

\begin{abstract}
A linguistic recursion refers to the ability of a phrase element to be nested (that is, dominated) into another element that contains the same category. Transformational generative grammar and systemic-functional linguistics both involve the form of "recursion", which is also explored in subsequent studies and analyses that go beyond "syntax" to "discourse structure". Taking traditional Chinese landscape painting as an example, the phenomenon of "recursion" often appears in artistic works. Therefore, in addition to logic, mathematics, linguistics and other disciplines, recursion is also reflected in the humanistic category of art, which is a universal dynamic mechanism in human thinking. The recursion of language and landscape painting is rooted in their structure and the holographic structure of the universe. This paper attempts to enumerate and discuss the similarities and differences between recursive phenomena in linguistic and artistic categories and grasp their basic laws and forms of change.
\end{abstract}

Keywords: recursion, embedding, systemic-functional syntax, discourse structure, landscape painting recursion phenomenon, universal structure congruence

\section{Recursion is studied from the perspective of syntax}

In linguistics, recursion refers to repetition or layers of nesting of the same structural components. In transformational generative grammar, Chomsky has put forward recursive devices, (Chomsky, 1957: 24), recursive processes (Chomsky, 1957: 23f), the recursive aspect, and the recursive tense system. But in Saussure's system there is no mention of the rulebound creativity involved in the everyday use of language. In a system of rules, you can build up some finite rules to produce an infinite number of sentences. It is not very difficult to do this and as long as a few recursive rules are used over and over again you will get a large number of sentences with relational clauses modifying nouns (see Liu Runqing, 1997: 110-111)

\section{Recursion is studied from the perspective of pragmatics}

Among the studies of discourse structure, narrative is the most widely studied. For example, the social linguistic model proposed by Labov was the first and had the greatest influence. Labov (1972) believed that a whole narrative structure consisted of the following six components: abstract + orientation + complicating action + evaluation + resolution + coda. Hoey $(1983,2001)$ put forward the structure model of discourse: situation — problem — response - evaluation. Hoey $(1983,2001)$ believed that this is the most common structure model of English discourse, and it can be used to describe and explain discourse structures in different domains, such as narration, advertisement and exposition (see Wang Yong, 2006: 289-290).

In fact, recursion is ubiquitous in discourse structures. The author finds that the two types of recursion discussed in functional syntax (linear recursion and embedded recursion) also exist in discourse structure: linear recursion and embedded recursion.

\subsection{Linear recursion in discourse structure}

For example, teaching exchange usually consists of three steps: (teacher) I, initiation, followed by (student) response (R), followed by (teacher) feedback (F). Initiation is an essential component of classroom communication. The latter two are optional (indicated by parenthesis). Therefore, the typical classroom communication structure can be expressed as: I (R) (F) (Sinclair \& Coulthard 1975: 26). According to Sinclair, communication can be regarded as the smallest textual unit in communication, which can be associated with basic structures such as initiation, response and feedback.

\subsection{Embedded recursion in discourse structure}

In narrative texts, stories are often embedded in stories. The story of one narrator is nested within the story of another 
narrator, and the loop repeats itself, nesting recursion indefinitely. For example, the play The Murder of Gonzago in the play Hamlet is a play within a play, that is, another story embedded in the whole narrative discourse.

Linear recursion and embedded recursion can be interwoven into the text, making the text more colorful, more complex and varied in structure. In theory, such linear recursion and embedded recursion can also be repeated indefinitely in the text, line by line, loop by loop.

\section{Summary of the recursion of syntax and discourse structure in linguistics}

The recursive structure of language is the same as the recursive structure of the universe. In other words, the subsystem (holographic element) is a rapid and brief replay of the parent system, and the two are holographic relations.

The recursion of the universe is described as follows, "The big universe contains countless small universes; the big system contains countless small systems; the high level contains countless low levels. Each small universe, low level and small system is the epitome of the big universe, high level and large system. We call the structure of 'Chinese box' holographic recursive structure" (Wang Cunzhen, Yan Chunyou, 1995: 54)

The whole language structure and speech generation are in the same structure of repetition and layer upon layer. The recursive nature of language is rooted in the recursive structure of the universe, the holographic law of the universe.

\section{Nesting and recursion in traditional Chinese landscape painting}

\subsection{Nature has the recursive feature of fractal as research and description object of traditional landscape painting}

In nature, many things or phenomena have the self-similar structure characteristic of fractal "recursion hierarchy", and such structure also exists on a larger scale in nature. Whether you scale it up or down at any size, the overall hierarchy doesn't change. The traditional Chinese landscape painting takes nature as the object of study and description, and its interlaced space, shape and ink form also reflect the character.

"The shape of a tree is reflected at different scales, and is also formed by a complex chaotic interaction of forces: the trunk forks into branches, the branches fork into twigs, and the leaves of the twigs repeat the pattern of the branches on the veins, with self-similar records in its large scale and small details from time to time" (John Briggs, David Peter (2004). In other words, the repeated patterns of the trunks, branches, branches, and veins of the leaves of nature in large and small details have "infinitely nested self-similar structures".

\subsection{The painting object of mountains and rivers in traditional Chinese landscape painting have the feature of recursion}

Chinese traditional landscape painters consider that mountains and rivers gave birth to the complex nature of all things in natural as well as object shape in nature. "The principle of the heaven and earth, the mass of the mountains and rivers" (Wang Bomin, Ren Daobin, 2002), and the traditional abstract landscape painting depict the extraction of "mass of mountains and rivers" to show "the principle of the universe". It also reflects the understanding of "tao". Huang Binhong: "Therefore, the mountains have their veins; the water has its source; the roads are winding and the trees have their roots". Truth comes from the change of nature (Wang Bomin, 1961). The mountains and valleys, forest springs trees, clouds, rain and fog, all inclusive, although complex, but still have the same basic shape and law of nesting copy.

For example, "small is stone, big is mountain", but mountain and stone have fractal similarity in different scales. Mountain and stone will look very similar; mountain is composed of countless similar stones in a certain regular way, forming nested recursive characteristics. For example, In Ni Zan's Rong Xi Zhai Tu (1372 Scroll ink on paper 74.7*35.5 $\mathrm{cm}$ in Taipei National Palace Museum), a leisurely shallow hill in the distance is a "mountain". Nearby simple stones are "stones". A group of stones and blocks can reflect the characteristics of the whole hill or even mountains and rivers. That is to say, the whole hill or mountains and rivers are recursive graphs obtained by the iteration of each stone layer. Such recursive shapes can also theoretically be superimposed, nested, and repeated indefinitely.

Another example is Fan Kuan's representative work Xishan Travel Map, which uses the strokes of "Rain-point Brushstroke" with rows of dotted lines. Chapped strokes represent the vertical texture and mass structure of mountain stone form. The chapped structure embedded in the overall image forms a recursive representation.

\section{The recursion of language and art coexists with the structure of the universe}

We can find from the whole language structure and speech generation, the object nature depicted by Chinese 
landscape painting, as well as the creation of Chinese landscape painting works and the works themselves. Recursion is a universal dynamic mechanism in human thinking. Both language and art are in the same structure of repetition and layer upon layer. The root of their recursion lies in their structure and the structure of the holographic universe.

\section{References}

[1] Chomsky N. Syntactic Structures. The Hague: Mounton \& Co.; 1957.

[2] Chomsky N. Aspects of the Theory of Syntax. Cambridge, Mass: The MIT Press; 1965.

[3] Halliday M A K. Class in relation to the axes of chain and choice in language. Linguistics. 1963; (2): 5-15.

[4] Labov W. Language in the Inner City. Philadelphia: University of Pennsylvania Press; 1972.

[5] Hoey M. On the Surface of Discourse. London: Allen and Unwin; 1983.

[6] Stubbs M. Discourse Analysis. Oxford: Blackwell; 1983.

[7] Liu Runqing. Western Schools of Linguistics. Beijing: Foreign Language Teaching and Research Press; 1997.

[8] Wang Yong, Huang Guowen. Recursion in Discourse Structure. Foreign Language Teaching and Research. 2006; 38(5): 288-295.

[9] Qian Guanlian. On Recursiveness of Language and Its Origin. Journal of Foreign Languages. 2001; (3): 8-14.

[10] Wang Cunzhen, Yan Chunyou. Cosmic Holographic Uni ication. Jinan: Shandong People's Publishing House; 1995.

[11] Benoit B. Mandelbrot. The Fractal Geometry of Nature. Translated by Chen Shouji, Ling Fuhua. Shanghai: Shanghai Far East Publishing House; 1998.

[12] John Briggs, David Peter. Seven Lessons in Chaos. Translated by Chen Zhong and Jin Wei. Beijing: Peking University Press; 2004.

[13] Wang Bomin, Ren Daobin. Painting Integration. Shijiazhuang: Hebei Fine Arts Press; 2002.

[14] Wang Bomin. Edited Quotations of Huang Binhong. Shanghai: Shanghai People's Fine Arts Publishing House; 1961. 